\title{
Compressions médullaires traumatiques : évolution du concept chirurgical
}

\author{
R. Roy-Camille \\ Service de Chirurgie Orthopédique et Traumatologique, Hôpital Pitié-Salpêtrière, 83, boulevard de l'Hôpital, F-75651 Paris Cedex 13
}

Dans le papyrus d'Edwin Smith écrit environ 3000 ans avant J.C., on trouve une description claire et précise de la tétraplégie par lésion traumatique du rachis cervical qui était déjà reconnue, et discutant le traitement, l'auteur, inconnu, dit qu'il s'agit à l'évidence d'une maladie qui n'a pas à être traitée.

Cette attitude vis-à-vis des compressions médullaires traumatiques s'est perpétuée pendant des milliers d'années sans changer.

Hippocrate, 400 ans avant J.C., entra dans une phase plus active et proposa la réduction des fractures sur son scammon ou cadre de réduction.

Ambroise Paré proposa la réduction des fractures en décubitus ventral et le maintien de la correction par des attelles et des plaques de plomb moulé (1564).

Paul d'Egine (625-690) semble avoir fait la première laminectomie décompressive.

Après les travaux de Lister et Pasteur, l'attitude chirurgicale s'est progressivement développée, basée uniquement sur la laminectomie mais jusqu'à la première guerre mondiale, les efforts du thérapeute en face d'une lésion médullaire complète étaient sans espoir. Durant cette guerre, Cushing rapporte dans l'armée américaine une mortalité de $80 \%$ dans les deux premières semaines, les lésions partielles ayant seules une chance de survie. La deuxième guerre mondiale ne changea d'abord rien au problème. Puis en février 1944, Ludwig Guttmann ouvrit le Centre de Stoke Mandeville.

La chirurgie qui consistait à l'époque uniquement en une laminectomie avait à juste titre une mauvaise réputation. Elle ajoutait le traumatisme opératoire - souvent inutile - ou traumatisme initial et n'était entourée d'aucun soin de nursing. La plaie opératoire rajoutait un souci aux complications de décubitus car on n'osait pas bouger les opérés après l'intervention. Guttmann établit un traitement conservateur prenant en charge le malade dès le début du traumatisme avec un nursing élaboré, précis et soigneux et le rendant ensuite à la vie au sein de la société avec une fonction active. Cette méthode fit merveille.

Mais Guttmann n'a pas toujours été compris. En effet, beaucoup ont ignoré qu'il ne demandait pas seulement de retourner les malades dans leur lit, mais qu'il réclamait aussi une réduction des fractures et luxations avec des coussins adaptés dans le lit. Je le cite : «Les luxations et les luxations-fractures des différents types, qu'elles soient stables ou instables, peuvent être réduites et stabilisées avec succès ». Il dit encore : «Je ne partage pas l'opinion que les simples fractures par compression ne nécessitent pas une réduction et une fixation ». Mais il ne voyait dans sa technique que le besoin de stabiliser la partie antérieure de la vertèbre pour éviter une cyphose secondaire. A le lire, on ne trouve jamais exprimé que la réduction rendait au canal rachidien sa forme normale et était de ce fait la meilleure décompression.

En Avril 1963, à Raymond Poincaré, dans le Service de Robert Judet, je fus confronté au problème. Une jeune fille de 17 ans, au cours d'un accident de voiture, avait subi une fracture-luxation de LA sur L5, L4 étant complètement déplacée sur le flanc latéral de L5, déplacement en baïon- nette et chevauchement. Cette lésion s'accompagnait d'un syndrome complet de la queue de cheval. Elle fut opérée en urgence dans un service de neurochirurgie, seuls services s'occupant alors des blessés médullaires à Paris et sans aucune réduction, on lui fit simplement une laminectomie décompressive de L4 et $\mathrm{L} 5$. $\mathrm{Au} 10 \mathrm{e}$ jour post-opératoire, cette patiente se retrouvant à Raymond Poincaré dans le Service de réhabilitation, je fus amené à l'opérer pour stabiliser le rachis. Je pus réduire facilement le déplacement par traction et manipulations sur table orthopédique, mais ne disposant plus ni d'épineuses, ni de lames, je ne pouvais pas utiliser les instruments dont on disposait à l'époque pour fixer le rachis, à savoir les plaques de Wilson qui se posaient sur les épineuses, ou les fils métalliques qui se posaient autour des lames. J'ai alors imaginé de mettre en place deux plaques de Shermann en fixant celles-ci au niveau des colonnes articulaires sur ce que je trouvais en face de moi. Je trouvai des prises plus ou moins fermes à mes vis. En post-opératoire, en comparant le montage avec un squelette du rachis que je pris en mains, je m'aperçus que là où il y avait de bonnes prises c'était dans les pédicules vertébraux et l'étude anatomique de ceux-ci me montra facilement que c'était le véritable bon point d'ancrage qu'il fallait chercher pour une vis dans le rachis. De cette observation, je concluais :

$1^{\circ}$ Que la laminectomie seule ne réalisait le plus souvent pas une bonne décompression.

$2^{\circ}$ Que par contre la réduction rendait au canal sa forme normale et 
était le geste le plus important pour lever la compression de l'axe nerveux central.

$3^{\circ}$ Qu'il pouvait être nécessaire d'enlever du canal un élément osseux ou discal compressif, la laminectomie devant alors être conçue comme une voie d'abord.

$4^{\circ} \mathrm{Qu}$ 'une stabilisation solide était nécessaire pour maintenir la réduction et pouvoir mobiliser les patients.

$5^{\circ}$ Que les pédicules étaient les meilleurs points d'ancrage pour implanter des vis qui soutiendraient les plaques de stabilisation.

$6^{\circ}$ Que les patients opérés nécessitaient les mêmes soins de nursing que les malades de Guttmann et la patiente repartit rapidement chez Monsieur Grossiord dans le centre de Rééducation.

Il fallut faire passer cette doctrine chirurgicale révolutionnaire dans les mœurs. Ce n'était pas facile à l'époque et il fallut beaucoup de patience et de persévérance. Cependant, les résultats s'améliorèrent et ceci d'autant que la réanimation faisait des progrès.

On vit ainsi la mortalité qui était aux environs de $80 \%$, et même pratiquement de $100 \%$ pour les traumatisés de plus de 50 ans, descendre dans une statistique présentée par Gallibert de 47,8 à $33,5 \%$ entre 1960 et 1967 et à $11,6 \%$ entre 1968 et 1970 . De même à Raymond Poincaré, nous voyions la mortalité passer de $20 \%$ entre 1963 et 1971 à $10 \%$ entre 1972 et 1976. Marie-Thérèse Cousin regroupant différentes statistiques publiait deux séries comparatives et sur 286 tétraplégiques opérés, la mortalité était de 17,83\%, tandis que sur 374 cas non opérés, la mortalité était de $34,76 \%$. Gallibert, en 1971, estimait que les aggravations ne dépassaient pas $5 \%$ chez les opérés alors qu'elles pouvaient représenter jusqu'à $13 \%$ en cas d'abstention thérapeutique ou de traitements purement orthopédiques. Les récupérations neurologiques s'avéraient aussi satisfaisantes. C'est ainsi que l'équipe de Garches
(Service du Pr R. Judet) rapportait en 1974 les chiffres suivants :

- 14 tétraplégies incomplètes opérées entre 0 et $24 \mathrm{~h}$ après le traumatisme présentaient 13 récupérations,

- 12 opérés entre 24 et moins de 48 h présentaient encore 7 récupérations.

C'est ainsi que depuis les années 80 , cette chirurgie de la compression médullaire a été de plus en plus adaptée :

- basée sur le principe de l'urgence mais en ne refusant pas le geste chirurgical à ceux qui arrivaient après un délai de 12 et voire de $24 \mathrm{~h}$;

- basée sur le principe de la levée de la compression par réduction de la fracture, qui rend au canal rachidien sa forme normale et libère ainsi la moelle; libération si besoin complétée par une laminectomie-voie d'abord, permettant l'extraction d'agents intrarachidiens; - basée aussi sur une fixation solide et stable évitant les déplacements secondaires, diminuant l'œdème au niveau du foyer de fracture et facilitant le nursing des malades en raccourcissant leur temps de séjour dans les centres de réhabilitation.

Au geste chirurgical qui garde toute sa valeur tel que nous venons d'en redéfinir les principes, s'ajoute maintenant un apport de la pharmacologie. Depuis 1990, l'utilisation de certaines drogues dans les $6 \mathrm{~h}$ qui suivent le traumatisme, expérimentalement et même cliniquement, semble améliorer le pronostic des contusions médullaires. Les publications à ce sujet se multiplient avec la méthylprednisolone qui prévient la mort cellulaire quand elle est utilisée à fortes doses en s'opposant à la peroxydation des lipides. Les lazaroïdes sont d'autres molécules, elles aussi dérivées des corticoïdes, ayant le même effet mais sans action gluco- ou minéralo-corticoïdes, ayant donc moins d'effets secondaires. Les phencyclydines inhibant les récepteurs membraneux au glutamate ainsi que les gangliosides qui bloqueraient le transport calcique et qui pourraient favoriser la croissance neuronale sont également à l'étude expérimentale. Toutes ces drogues sont censées prévenir la mort cellulaire par les différents mécanismes indiqués.

Cette voie pharmacologique sera sûrement des plus utiles, mais en sachant que les drogues doivent être administrées très tôt après le traumatisme. Leur utilisation n'enlève rien au geste chirurgical.

Dans ce domaine, d'autres travaux sont en cours et peut-être que des greffes cellulaires corticales embryonnaires pourront tenir certaines promesses expérimentales selon les résultats obtenus par Gopal Das.

\section{Bibliographie}

1. Cousin MT, Bozier A (1978) Problèmes posés à l'anesthésiste-réanimateur par les traumatismes du rachis cervical. In : Les urgences en anesthésie et réanimation. Librairie Anette, Paris, pp 287-304

2. Gallibert $P$, Grunewand $P$, Delcour $J$, Rosat $P$ (1971) Le traitement des traumatismes du rachis cervical compliqués par des lésions médullaires ou radiculaires. Optique neurochirurgicale. Etude critique de 300 cas de traumatismes rachidiens et médullaires cervicaux observés en 20 ans. Ann Med Phys 14: 334-371

3. Guttmann L (1973) Spinal cord injuries. Comprehensive management and research. Blackwell

4. Judet R, Honnart F, Senly G, Piera JP (1974) Lésions du rachis cervical avec troubles neurologiques. In : Actualités de chirurgie orthopédique de l'Hôpital Raymond Poincaré. Masson, Paris, pp 97-122

5. Pare A (1585) Euvres complètes, 4e edn. Gabriel Buon, Paris, 4e éd

6. Roy-Camille et coll. (1983) Rachis traumatique neurologique. In : 3es Journées d'Orthopédie de la Pitié. Masson, Paris, pp 151-205

7. Roy-Camille R, Mazel Ch, Zeller R, Edouard $\mathrm{B}$, Lapresle $\mathrm{Ph}$ (1988) Traumatismes du rachis cervical. In : Lésions avec atteinte médullaire. In : 6es Journées d'Orthopédie de la Pitié. Masson, Paris, pp 120-125

8. Roy-Camille R, Zerah JCl (1970) Ostéosynthèse des fractures du rachis dorsal et lombaire. In : 8es Actualités de Chirurgie Orthopédique de l'Hôpital Raymond Poincaré. Masson, Paris, pp 196-203 